FACTA UNIVERSITATIS

Series: Mechanical Engineering Vol. 16, No 1, 2018, pp. 65 - 75

https://doi.org/10.22190/FUME180109011C

Original scientific paper

\title{
THE "SPORT" OF ROUGH CONTACTS AND THE FRACTAL PARADOX IN WEAR LAWS
}

\author{
UDC 539.6
}

\begin{abstract}
Michele Ciavarella ${ }^{1}$, Antonio Papangelo ${ }^{1,2}$
${ }^{1}$ Department of Mechanics, Mathematics and Management, Politecnico di Bari, Italy ${ }^{2}$ Department of Mechanical Engineering, Hamburg University of Technology, Germany
\end{abstract}

\begin{abstract}
In a recent paper in Science, namely, "The Contact Sport of Rough Surfaces", Carpick summarizes recent efforts in a "contact challenge" to predict in detail an elastic contact between the mathematically defined fractal rough surfaces under (very little) adhesion. He also suggests the next steps that are needed to "fulfill da Vinci's dream of understanding what causes friction". However, this is disappointing as friction has been studied since the times of Leonardo and in 500 years, no predictive model has emerged, nor any significant improvement from rough contact models. Similarly, a very large effort we have spent on the "sport" of studying rough surfaces has not made us any closer to being able to predict the coefficient of proportionality between wear loss and friction dissipation which was already observed by Reye in 1860. Recent nice simulations by Aghababaei, Warner and Molinari have confirmed the criterion for the formation of debris of a single particle, proposed in 1958 by Rabinowicz, as well as Reye's assumption for the proportionality with frictional loss, which is very close to Archard anyway. More recent investigations under variable loads suggest that Reye's assumption is probably much more general than Archard's law. The attempts to obtain exact coefficients with rough surfaces models are very far from predictive, essentially because for fractals most authors fail to recognize that resolution-dependence of the contact area makes the models very ill-defined. We also suggest that in the models of wear, rough contacts should be considered "plastic" and "adhesive" and introduce a new length scale in the problem.
\end{abstract}

Key Words: Rough Contact, Fractals, Adhesive Wear, Reye's Law, Archard's Law, Rabinowicz' Criterion

Received January 09, 2018 / Accepted February 07, 2018

Corresponding author: Michele Ciavarella

Department of Mechanics, Mathematics and Management, Politecnico di Bari, Viale Japigia 182, 70126 Bari, Italy

E-mail: mciava@poliba.it 


\section{INTRODUCTION}

Of all the basic tribological phenomena of contact, adhesion, friction, lubrication and wear, very few have really benefited from the detailed studies many academics are putting into exploring rough contacts. In a recent comment in Science, namely, "The Contact Sport of Rough Surfaces" [1], Carpick has summarized a recent competition where "approximate models of interacting surfaces competed against a supercomputer solution", and concluded that "the multiscale approaches had the winning score". Also that, although multiscale models were already invented by Archard [2], "the time has come to standardize multi-scale descriptions with elastic coupling", providing, however, no experimental evidence that this effect of elastic coupling is really so important in any tribological model.

One of the basic laws of friction dictates that "force of friction is independent of the apparent area of contact" but was already noticed by Leonardo da Vinci although not properly published at a time. Do we have any model today that can predict the friction coefficient, based on the rough surface details? On the contrary, despite very large attempts to correlate friction coefficient, including roughness, no clear formula is known. Perhaps some effects of roughness on friction in viscoelastic materials are understood qualitatively, but basically they correspond to a single scale of roughness, and they are extremely sensitive to the so-called large wavevector cutoff, which remains rather arbitrary [3].

Archard's model did predict the linearity of the contact force with the real contact area already in 1953, and at that time, this was a real innovation. In fact, he even anticipated "fractals" which were much later used in a more refined form in the "contact sport" of rough surfaces. Yet it was only much later that Ciavarella et al. [4] remarked that linearity involves a linear coefficient which is scale-dependent, and in particular it goes to zero for realistic fractal geometry. This was done with the not very popular choice of a Weierstrass series as a fractal, but later Gaussian model theories [5] did not change the basic conclusion that "no applied mean pressure is sufficiently large to ensure full contact and indeed there are not even any contact areas of finite dimension - the contact area consists of a set of fractal character for all values of the geometric and loading parameters". Hence, the contact area was found to have a (limiting) fractal dimension of (2-D), where D is the fractal dimension of the surface profile. The idea [5] of a "magnification"-dependent solution is rather a mathematical trick for avoiding the discussion of an "ill-defined" nature of the contact area.

Wear remains the least scientifically understood tribological process. The most common approach in wear refers to Archard [2] as wear volume $V$ is proportional to sliding length $L$, normal force $W$, and inverse with hardness $H$ of the material [6]. Hence, wear rate $V^{\prime}$ is proportional to pressure $p$ via a wear coefficient $K$ :

$$
V^{\prime}=\frac{K p}{H}
$$

This proportionality is satisfied for many pure metals, see Ref. [7], but more in general, there is a more complex dependence (see Fig.1), in some cases even contradicting the Archard wear law inverse dependence on hardness, especially for very high hardness. The latter result may be connected to Kragelsky's [8] observation that catastrophic wear occurs when surface layers are harder than substrate, whereas the opposite is suggested for minimizing wear (see also Popov et al. [9]). Therefore, even just looking at Fig.1 reveals 
that there is more in $\mathrm{k}$ than what "wear coefficient" constant suggests. It probably depends on other factors, and indeed, [7] suggests there are two ranges: in Range I the wear coefficient is constant and low, and the wear resistance increases proportionally to hardness. Instead, in range II, the wear rate can either increase (but less than in Range I), or be independent of, or decrease with hardness depending on the particular toughness.

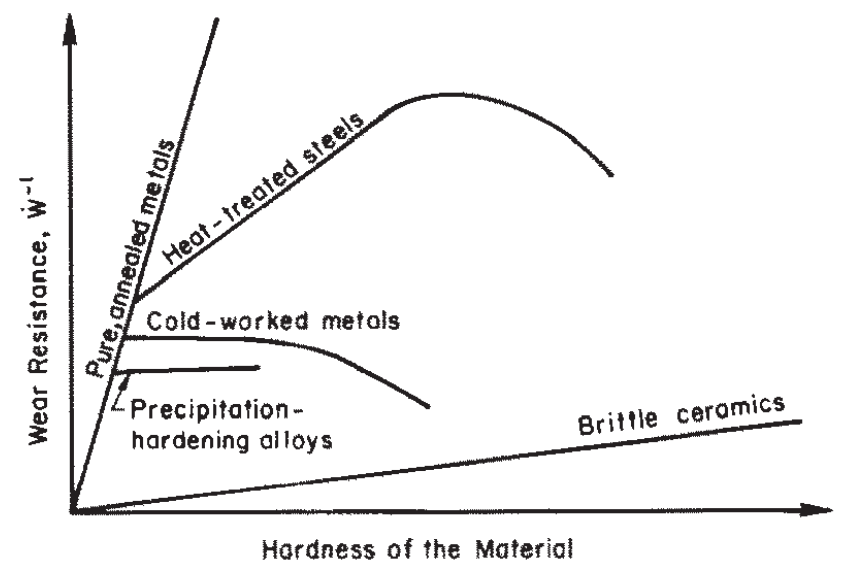

Fig. 1 Some example dependencies of wear resistance for various materials (from [7])

Hence, while the influence of hardness is not rigorous in the Archard law, the proportionality with normal load is more robust and indeed it was proposed much earlier in Europe by Reye [10] in a rather unfortunately unknown paper (it has 50 citations in Google Scholar, which in the days of bibliometric assessments of research impact, should mean it is a rather bad paper!). In Italy, for example, Reye's assumption is universally suggested in undergraduate engineering course of brakes design at least after the II World War, and it is usually put in terms of wear rate being "proportional to frictional work expenditure". Before continuing our discussion, an interesting paper to cite is a very recent one by Akbarzadeh \& Khonsari [11]. They investigated pin-on-disk experiments with a variable loading sequence. The experimental results show that the loading sequence affects weight loss (contradicting another old known law in fatigue, Palmgren-Miner), which correlates well with the dissipated power, regardless of the loading sequence, while Archard's law was shown to be inaccurate. Hence, Reye's assumption is probably more general than Archard's.

The general picture remains confused, and there is no alternative to measurements. Meng and Ludema [12] confirm, after a detailed analysis, that no single predictive equation or group of limited equations could be found for general and practical use in wear, and suggest that the "reasons include the perpetuation of erroneous and subjective expressions for the mechanisms of wear", and "the paucity of good experiments to verify proposed models". We suspect that this situation will continue for many more attempts to propose miraculous predictive equations for wear. 


\section{The RABinowicz CRitical Length SCALE}

One of the interesting ideas for adhesive wear (one of the most prevalent types of wear) was suggested in 1958 by Rabinowicz [13] in Wear and was later forgotten (the paper has 12 citations in Google Scholar, even worse than Reye's paper [10]), distinguishing the regimes of plastic smoothing and the formation of wear particles in a contact of homogeneous sliding bodies. Its summary says that "whether a fragment transferred during sliding comes off as a loose wear particle is shown to depend on whether its elastic energy exceeds the surface energy at its point of attachment. Calculations show that, for an equiaxed fragment, there is a critical size, such that smaller fragments remain adherent while larger fragments come off in loose form. Experimental support for this critical size concept is cited. The material parameters involved are such that smaller fragments are formed with hard metals than with soft metals, thus explaining the better surface finish during sliding observed with the former". There was considerable experimental evidence in support of the criterion, as for example, in the tests involving steel sliding on steel,with only the largest fragment formed (diameter $120 \mu \mathrm{m}$ ) "could be loosened from the surface to which it adhered by gentle mechanical treatment". In a recent paper, Aghababaei et al. [14] have confirmed by explicit mesoparticle simulation, that the transition does exist so strongly supporting to general Rabinowicz's competition of plastic deformation and fracture. The Rabinowicz-Aghababaei length scale is more in details

$$
a^{*}=\lambda G \frac{w}{\sigma_{j}^{2}}
$$

a function of shear modulus $G$, the fracture energy per unit area $w$ and junction strength $\sigma_{j}$ with a shape factor $\lambda$.

Therefore, the basic ideas for wear return regularly but were formed already a long time ago: the novelty is that with new simulation techniques, it is tempting to think that one could be perhaps able to "predict" wear coefficients, which are otherwise experimentally widely differing by more than 7 orders of magnitude. To explain the concept of wear coefficient we need to mention Archard who proposed that "we postulate: worn volume $\sim a^{3}$ and effective sliding distance $\sim \mathrm{a}$, therefore, the contribution of this contact to the wear per $\mathrm{cm}$ of sliding $\sim a^{2}$; also load supported by contact $\sim a^{2}$. Therefore, for this contact, the contribution to the wear rate is proportional to the load supported by it. A similar argument applies to all other contacts, and the total wear rate is proportional to the load". He had in mind, of course, the plastic junctions of Bowden and Tabor [15], for which the real contact area is obviously proportional to load, via hardness. Hence, the proportionality of wear rate with normal load is known almost universally as the "Archard wear law", because of the hardness dependence, which we have seen is not so universal. The linearity with load also occurs under elastic regime (as proved also by Archard in his multiscale model), with an important difference that in this case there is no reference to hardness. However, Archard's explanation for his law is unconvincing in that he assumes a constant density of asperities with height, which is universally known to be not a reasonable assumption of a real surface and more importantly, all asperities are wearing in his model, in contrast with the Rabinowicz-Aghababaei critical size concept. Instead, Reye's assumption, proportionality of wear with frictional work, has also been confirmed again by very nice modern simulations in small systems by Aghababaei et al. [16]. 


\section{Multiscale Contact AtTempts to Derive Wear CoefFicient AND THE FRACTAL PARADOX}

Despite our concern that the Archard law is inevitably connected to the concept of hardness, and the latter is strongly connected to plastic deformations, recent attempts are emerging to infer wear coefficients by upscaling the concept of the Rabinowicz length scale in multiscale contact. Frérot et al. [17] for example inserted the RabinowiczAghababaei critical scale in the Archard model, i.e. assumed $K$ is the probability that contact area $A$ is larger than $A^{*}$ (where $A^{*}$ is the area of critical length scale $a^{*}$ ) for a given load $W$

$$
K=P\left(A>A^{*}\right)=\int_{A^{*}}^{\infty} p(A, W) d A
$$

and found common characteristics with experimental observations. More precisely, starting from analytical predictions, using the quasi-realistic Greenwood-Williamson model with exponential distribution of asperities $\varphi=\left(C / \sigma_{s}\right) \exp \left(-z_{s} / \sigma_{s}\right)$ for $z_{s}>0$, and $\sigma_{s}$ being a scale parameter of the order of RMS amplitude, $p(A, W)$ turns out a negative exponential independent of applied load $W$

$$
p(A, W)=\frac{1}{b \sigma_{s}} \exp \left(-\frac{A}{b \sigma_{s}}\right)
$$

where $b=\pi R$ for elastic, and $b=2 \pi R$ for plastic model.

Therefore, (i) wear coefficient $K$ is also independent of the load,

$$
K=\exp \left(-\frac{A^{*}}{b \sigma_{s}}\right)
$$

Also, in this model (ii) there is a linear relationship between the contact area and the applied load in both elastic and plastic cases. Both (i) and (ii) observations are consistent with experimental observations. $K$ values reported in experiments are in the range $10^{-8}$ $10^{-1}$, and there are too many constants in the model to judge if the prediction is realistic or not. Notice the dependence on the Rabinowicz length scale but also that on roughness amplitude as for lower roughness lower wear rate is predicted. One aspect that is not discussed, however, in [17] is the dependence on radius. Also a lower radius shows a lower wear rate, and given that the sensitivity to radius to resolution of the instrument (or to truncation of the fractal process) is extremely high, this would mean that the elastic model predicts always infinitesimally small wear! We can add this to many rough contact paradoxes, which are nevertheless often forgotten, when attempting to model precisely the trend towards meaningless results.

Notice that this is not a consequence of the simple asperity model. Lately, the academic desire to improve models has led to a large effort all over the world to improve elastic models with fractal surfaces, as we have discussed in the opening of the paper, so that Carpick calls this "contact sport" [1, and see Refs. therein]. This is due to the fact that it is much more difficult to include plasticity in models rather than refining asperity models. In fact, more refined models in [17] with more "exact" BEM numerical calculations show that the probability density function of the cluster areas follows a negative power-law with exponent 1.5 in a certain interval of $A$, where it is independent 
of $W$, up to a upper bound value $A_{m}$ which increases with the load. This results in small differences with respect to the simple and analytical Archard-GW exponential model: namely, the wear coefficient transitions from zero (i.e. no observable wear) to a constant value. This constant value decreases if $A^{*} / \lambda_{s}{ }^{2}$ increases, where $\lambda_{s}$ is the smallest wavelength in the roughness spectrum. This dependence (which is the counterpart of the dependence on the radius of the GW model) is not fully discussed in [9] but obviously means that the truncation of the fractal process is a very sensitive parameter also in the "refined" BEM models, as in the fractal limit, $\lambda_{s} \rightarrow 0$ or $R \rightarrow 0$ and there is no longer predicted wear.

The returning problem as we have discussed recently in [18] is that the "real contact area" is often an ill-defined, "magnification" dependent quantity. In the Bowden-Tabor view, which was a plastic model, later used by Archard to derive his hardness dependent law, this problem obviously does not exist. Of course, macroscopic quantities like stiffness or load vs. separation, are not so magnification dependent, and on these properties, elastic models can be used.

Finally, notice that another (more ambitious) interpretation of the wear coefficient in [17] based on an alternative assumption, namely that wear coefficient $\mathrm{K}$ would be equal to the ratio between the area of contact satisfying the Rabinowicz-Aghababaei critical scale criterion, to the total real contact area, ie. not making the Archard connection with the probability of wear particle formation shows features in contrast with experiments, and here it is disregarded.

Summarizing, the analytical Archard-GW exponential model with the RabinowiczAghababaei critical scale concept has qualitatively correct features, and could be further considered for other investigations, provided we knew the scale for truncation of the fractal process. One can obviously decide this scale is universal and the reason in this regard. But it may well be quite a strong assumption.

\section{PLASTICITY MODELS}

The discussion in the paper so far, and the very nature of the Archard law, with its hardness in the denominator, highly suggest that a plastic model should be used in any attempt to extrapolate wear coefficients. In an attempt to solve the paradoxes of Ciavarella et al. [4], Gao et al. [19] added plasticity in the Weierstrass model. While the elastic model [4] showed that the "fractal limit" consists of an infinite number of contact spots, with zero size, subjected to infinite contact pressure, Gao et al [19]'s model found that while the total contact area and contact pressure are well defined, it remains impossible to predict the actual number of contacts or their size. They also suggest to add adhesion (but the problem with both plasticity and adhesion has not even been attempted yet) or truncating the fractal process where the fractal description breaks down. This is indeed typically done in numerical simulations such as those described in "contact challenge" exercises described in [1], but in that case the truncation is rather arbitrary, and not many authors make even an attempt to discuss how they make this truncation.

On the other hand, this poses some questions about the use of elastic simulations. Gao et al. [19]'s model shows that, for coarse resolutions, the elastic model is a good description of the full solution. Hence, it becomes important to look at the 3 dimensionless parameters defined by Gao et al. [19], which in practice essentially depend on wavelength scale parameter and amplitude parameter in the power spectrum density (continuous approximation) of the 
Weierstrass profile, the elastic modulus and the yield strength. Therefore, the results of the previous paragraph, where radius and amplitude of roughness entered directly into the prediction of the $\mathrm{K}$ wear coefficients, would need to be modified only if the resolution at which the problem is looked is increased enough to see plasticity effects, and this depends on an additional set of parameters. If the plastic regime is relevant, then the constants involved are the same, but in a different mix.

Notice also that the results of Gao et al. [19] have been compared with much more sophisticated predictions with detailed finite element simulations of Pei et al. [20], and show very similar features, for example the predicted true contact area as a function of the ratio of yield stress to elastic modulus. In Gao's calculations, the elastic-plastic transition (the elastic regime) disappears. Pei et al. [20], however, report more in details that plasticity produces qualitative changes in the distributions of the connected contact regions, with large clusters becoming more likely. However, as seen in Fig. 2, the elastoplastic model shows that the distribution of contact clusters is much closer to the very simple overlap model than to the elastic one. Once again, decades of discussion about elastic models (the "contact sport" of rough surfaces) seem largely unjustified. We hope at least that in the future, simplicity will guide our efforts rather than competitions over precise results of mathematical problems of little real tribological interest.

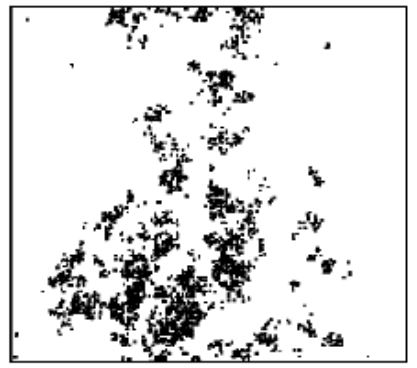

a)

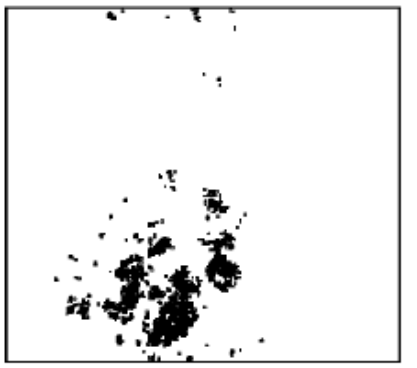

b)

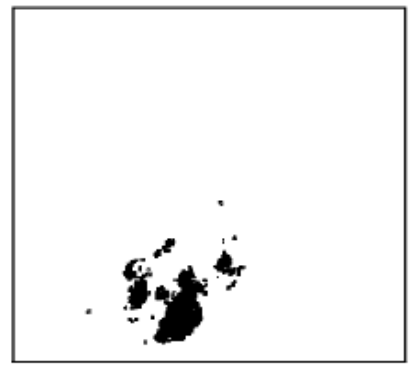

c)

Fig. 2 An example of contact area prediction for: a) elastic; b) elasto-plastic and c) rigid overlap model (adapted from [20])

\section{ADHESION MODELS}

In the paper so far, and in the review of previous models, an important ingredient for "adhesive wear" is surprisingly missing. They do not consider that at the contact interface the average size of the micro contacts will strongly depend on the strength of adhesion, which is a contradiction to the idea that plasticity junction should be formed in the first place, that is before possibly breaking in a wear particle.

The Rabinowicz criterion is based on consideration of competition of adhesion and plasticity. Analysis carried out in [21] illustrates that the Rabinowicz criterion is valid even if this competition occurs directly in the same interface. In most of the most previous works, the size of asperity is assumed as given and independent of adhesion. However, in an adhesive contact of curved surfaces, the contact size does depend on adhesion, too. In this Section we discuss this aspect of the problem. Let us note that there 
also exist other approaches where the poorly defined notion of an asperity is avoided [9, 22]. However, even within such a concept, the effective work of adhesion (which is proportional to the real contact area, which is also an ill-defined quantity [18]) does play a central role, and this area is also dependent on adhesion.

For the limited scope of the present paper, we shall attempt an elementary step towards a more complete picture, adding adhesion at the interface.

In the presence of adhesion, the standard JKR equation [23, 24] gives the contact radius a for a given normal force $P$,

$$
P=\sqrt{8 \pi E^{*} a^{3} w}-\frac{4 E^{*} a^{3}}{3 R}
$$

where $1 / E^{*}=1 /\left[E_{1} /\left(1-v_{1}^{2}\right)\right]+1 /\left[E_{2} /\left(1-v_{2}^{2}\right)\right]$ and $E_{i}, i=1,2$, are Young's moduli and $v_{i}, i=1,2$, Poisson's ratios of each material.

In other words, contacts cannot be smaller than these sizes, respectively upon approach, and before detachment. Moving, therefore, to a multiasperity contact, we disregard the Archard's uniform multi-asperity contact model as unjustified geometrically, despite its leading to reasonable results. Indeed, surfaces are today considered to be approximately Gaussian. An exponential tail is, however, a reasonable assumption, for qualitative purposes.

Consider a density of asperities $N$ and $f, g$ the functions relating area and load for each asperity, to its compression $\delta$, which is here given by the height of the asperity minus the separation between mean planes $\left(z_{s}-u\right)$.

The exponential distribution of heights permits to elucidate some results very clearly in the present context, obtaining the number of asperities in contact, total area $A$, and load $P$, as

$$
\begin{aligned}
& n=C N \exp \left(-\frac{u}{\sigma_{s}}\right) \int_{\delta_{1}}^{\infty} \exp \left(-\frac{\delta}{\sigma_{s}}\right) d\left(\frac{\delta}{\sigma_{s}}\right) \\
& A=n \int_{\delta_{1}}^{\infty} f\left(\frac{\delta}{\sigma_{s}}\right) \exp \left(-\frac{\delta}{\sigma_{s}}\right) d\left(\frac{\delta}{\sigma_{s}}\right) \\
& P=n \int_{\delta_{1}}^{\infty} g\left(\frac{\delta}{\sigma_{s}}\right) \exp \left(-\frac{\delta}{\sigma_{s}}\right) d\left(\frac{\delta}{\sigma_{s}}\right)
\end{aligned}
$$

where $\delta_{1}=0$ in the phase of loading, because JKR by assumption takes contact only when there is intimate contact, but $\delta_{1}=\delta_{0}$ upon unloading. Irrespective of whether we are in the phase of loading or unloading, it appears clear that area $A$ and load $P$ are proportional to each other, as in the classical repulsive case, and regardless of the JKR assumption. Notice in fact that these results hold also for plastic and any other constitutive law within the approximation of asperities, of course.

Hence, if we do consider the JKR solution (see Appendix for details), if $a_{\text {in }}>a^{*}$

$$
a_{i n}=\frac{R}{\left(\frac{E^{*} R}{w}\right)^{1 / 2}} \hat{a}_{i n}=(2 \pi)^{\frac{1}{3}}\left(\frac{w}{E^{*}}\right)^{\frac{1}{3}} R^{\frac{2}{3}}>\frac{\lambda G w}{\sigma_{j}^{2}}
$$


then the entire distribution of asperities will wear, and this will be proportional (or nearly proportional) to load. Therefore, we retain the main experimental observations known as the Archard law.

For simplicity, we are using, of course, the same surface energy which enters in the interface problem also in the detachment of wear particles -- as a reasonable first guess -and otherwise it is trivial to generalize the model keeping two separate material properties. Also, to make qualitative assessment, we assume that only one of the bodies is wearing out, the softer one which has also a smaller elastic modulus so that we can consider $E^{*} \approx$ $E$. Therefore, we can consider $G=E /[2(1-v)]$

$$
R>w \frac{E^{2}}{\sigma_{j}^{3}}\left(\frac{\lambda}{2(2 \pi)^{1 / 3}(1+v)}\right)^{3 / 2}
$$

As $\sigma_{j} / E=3 \cdot 10^{-3}$ for many materials, assuming $\lambda=1$

$$
R>4 * 10^{6} \frac{w}{E}
$$

For a crystalline material, $w / E=0.05 a_{0}$ where $a_{0}$ is atomic spacing, of the order of less than a nanometer; therefore, we can estimate very crudely $w / E \approx 10^{-11} \mathrm{~m}$, and the condition becomes

$$
R>40 \mu \mathrm{m}
$$

Naturally, under this condition, all the asperities will wear out, and the $K$ coefficient will take an extremely unlikely value of one. It is, therefore, unlikely that this condition provides anything very useful in practice. It is more likely to be another of the possible paradoxical results of applying multiscale contact models to Archard and Rabinowicz's ideas. However, notice that the paradoxical behavior occurs here not in the fractal limit, but rather on the contrary, if the resolution is not increased enough for asperity radii to decrease below this value.

Obviously, if the condition (11) is not fulfilled, one would have to integrate the asperity radii greater than the Rabinowicz Aghababaei length scale, similarly to what is done in [17]. The conclusion would simply be that for small $A^{*}$, wear coefficient $\mathrm{K}$ will remain unity until the condition (11) becomes violated, and then it would start to decrease, vaguely similarly to the adhesionless counterpart of [17]. Actually, we can predict that for large $A^{*}$, wear coefficient $K$ will tend asymptotically exactly to the adhesionless one, since for large contact areas the load is also large and the adhesive correction is likely to be small.

\section{CONCLUSIONS}

In the present paper, we have discussed that the contact "sport" of simulating an elastic multiscale contact with fractal accurate models that has dominated part of the specialized literature, is still very remote from solving any actual real tribological problem as prediction of friction coefficient or, even worse, wear coefficient. We have shown that many classical ideas that return periodically have been also confirmed in modern ways by numerical simulations. However, the most promising form of wear law 
is Reye's assumption rather than Archard's, at least according to some recent experiments under a variable amplitude load. Archard's models are often surprisingly discussed assuming no plasticity, clearly despite the hardness term in the denominator; it calls for an elasto-plastic analysis, and in this respect we have not made much progress since the qualitative ideas of Reye, Archard, Bowden-Tabor. Also, the "adhesive" wear law calls strongly for adhesion, and yet no model so far has ever discussed the effect of adhesion. We have attempted some qualitative discussion of these two aspects, and, concerning adhesion, we have effectively introduced another critical length scale in the problem, which is the minimum size of adhesive contacts based on JKR theory [23, 24]. The effect of adhesion is as intuitively expected, that of increasing the contact areas, and hence the wear coefficient with respect to the non-adhesive case. However, at least within the exponential approximation, the normal load and the area remain proportional. Also for some geometries, and combination of material properties, the effect would seem to obtain wear coefficients of unity; in particular, we write this in terms of an upper bound to the radius of asperities, above which this very high wear is predicted. As we estimate this upper bound to be quite high (hundreds of microns) but not incompatible with worn particle size measurements, we find reason for possible further discussion.

Acknowledgements: The authors thank R. Aghababaei and V. Popov for useful discussions and for sharing preprints of their submitted papers. A.P. is thankful to the DFG (German Research Foundation) for funding the project HO 3852/11-1.

\section{REFERENCES}

1. Carpick, R.W., 2018, The contact sport of rough surfaces, Science, 359(6371), pp. 38-38.

2. Archard, J.F., 1953, Contact and Rubbing of Flat Surfaces, Journal of Applied Physics, 24, pp. 981-988.

3. Ciavarella, M., 2018, A simplified version of Persson's multiscale theory for rubber friction due to viscoelastic losses, Journal of Tribology, 140(1), 011403.

4. Ciavarella, M., Demelio, G., Barber, J.R., Jang, Y.H., 2000, Linear elastic contact of the Weierstrass profile, In Proceedings of the Royal Society of London A: Mathematical, Physical and Engineering Sciences, Vol. 456, No. 1994, pp. 387-405.

5. Persson, B.N., 2001, Theory of rubber friction and contact mechanics, The Journal of Chemical Physics, 115(8), 3840-3861.

6. Dimaki A.V., Dmitriev, A.I., Menga, N., Papangelo, A., Ciavarella, M., Popov, V.L., 2016, Fast HighResolution Simulation of the Gross Slip Wear of Axially Symmetric Contacts, Tribol. Trans., 59, pp. 189-94.

7. Hornbogen, E., 1975, The role of fracture toughness in the wear of metals, Wear, 33(2), pp. 251-259.

8. Kragelski, I.V., 1965, Friction and Wear, Butter Worth.

9. Popov, V.L., Gervé, A., Kehrwald, B., Smolin, I.Y., 2000, Simulation of wear in combustion engines, Computational Materials Science, 19(1), pp. 285-291.

10. Reye, Th., 1860, Zur Theorie der Zapfenreibung, J. der Civilingenieur., 4, pp. 235-255.

11. Akbarzadeh, S., Khonsari, M.M., 2016, On the Applicability of Miner's Rule to Adhesive Wear, Tribology Letters, 63(2), pp. 1-10.

12. Meng, H.C., Ludema, K.C., 1995, Wear models and predictive equations: their form and content, Wear, 181, pp. 443-457.

13. Rabinowicz, E., 1958, The effect of size on the looseness of wear fragments, Wear, 2, pp. 4-8.

14. Aghababaei, R., Warner, D.H., Molinari, J.-F., 2016, Critical length scale controls adhesive wear mechanisms, Nature Communications, 7, 11816

15. Bowden, F.P., Tabor, D., 2001, The Friction and Lubrication of Solids, Clarendon Press.

16. Aghababaei, R., Warner, D.H., Molinari, J.-F., 2017, On the debris-level origins of adhesive wear, Proceedings of the National Academy of Sciences, 114(30), pp. 7935-7940.

17. Frérot, L., Aghababaei,R. Molinari, J.F., 2018, On the understanding of the wear coefficient: from single to multiple asperities contact, submitted, personal communication. 
18. Ciavarella, M., Papangelo, A., 2017, Discussion of "Measuring and Understanding Contact Area at the Nanoscale: A Review" (Jacobs, TDB, and Ashlie Martini, A., 2017, ASME Appl. Mech. Rev., 69(6), p. 060802), Applied Mechanics Reviews, 69(6), 065502.

19. Gao, Y.F., Bower, A.F., 2006, Elastic-plastic contact of a rough surface with Weierstrass profile, Proceedings of the Royal Society of London A: Mathematical, Physical and Engineering Sciences, 462(2065), pp. 319-348.

20. Pei, L., Hyun, S., Molinari, J.F., Robbins, M.O., 2005, Finite element modeling of elasto-plastic contact between rough surfaces, Journal of the Mechanics and Physics of Solids, 53(11), 2385-2409.

21. Popov, V.L., 2017, Generalized Rabinowicz'criterion for adhesive wear for elliptic micro contacts, AIP Conference Proceedings, 1909 (1), 020178.

22. Li, Q., Popov, V.L., 2018, On the possibility of frictional damping with reduced wear: A note on the applicability of Archard's law of adhesive wear under conditions of fretting, Physical Mesomechanics, 21(1), pp. 94-99.

23. Johnson, K.L., Kendall, K., Roberts, A.D., 1971, Surface Energy and the Contact of Elastic Solids, Proceedings of the Royal Society of London, Series A, 324, pp. 301-313.

24. Popov, V.L., Heß, M., 2015, Method of dimensionality reduction in contact mechanics and friction, Springer, Berlin Heidelberg.

\section{APPENDIX - ON JKR EQUATIONS}

We introduce the following dimensionless notation for the approach, and the load in spherical contact

$$
\hat{a}=\frac{a}{R} \beta ; \hat{\delta}=\frac{\delta}{R} \beta^{2} ; \hat{P}=\frac{P}{\pi R w}
$$

where $\beta=\left(E^{*} R / w\right)^{1 / 3}$. The JKR theory gives

$$
\hat{\delta}=\hat{a}^{2}-\sqrt{2 \pi \hat{a}} ; \quad \hat{P}=\frac{4}{3} \hat{a}^{3}-\sqrt{8 \pi \hat{a}^{3}}
$$

The JKR theory can be presented in a curve fitted form indistinguishable from the actual JKR curve, in order to be easily used in terms of $\hat{\delta}$

$$
\hat{P}=\hat{P}_{0}-1.1\left(\hat{\delta}-\hat{\delta}_{0}\right)^{1 / 2}+0.43\left(\hat{\delta}-\hat{\delta}_{0}\right)^{3 / 2}
$$

where $\hat{\delta}_{0}=-(3 / 4) \pi^{2 / 3}, \hat{P}=-5 / 6$ are the JKR values at pull-off in displacement control. To obtain the contact radius as a function of displacement which is easier to use in asperity models, we find a good best-fit as

$$
\hat{a}=4.594 \cdot 10^{-6} \hat{\delta}^{2}+0.9\left(\hat{\delta}-\hat{\delta}_{0}\right)^{1 / 2}+8.698 \cdot 10^{-3}\left(\hat{\delta}-\hat{\delta}_{0}\right)-3.342 \cdot 10^{-4}\left(\hat{\delta}-\hat{\delta}_{0}\right)^{3 / 2}
$$

These equations show an important feature. There is a jump-in to contact situation, where the surfaces approach each other, and jump-out of contact which this depends on the control, but we shall assume that for a multiasperity contact, displacement control is more realistic given we assign the displacement

$$
\hat{a}_{\text {in }}=(2 \pi)^{1 / 3} ; \quad \hat{a}_{\text {out }}=\frac{\pi^{1 / 3}}{2}
$$

